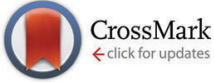

Cite this: J. Mater. Chem. C, 2016, 4,8822

Received 17th June 2016, Accepted 26th August 2016

DOI: $10.1039 / c 6 t c 02502 a$

www.rsc.org/MaterialsC

\title{
Excitonic quantum confinement modified optical conductivity of monolayer and few-layered $\mathrm{MoS}_{2} \dagger$
}

\author{
Guang Yi Jia, ${ }^{\text {ab }}$ Yue Liu, ${ }^{a}$ Jing Yu Gong, ${ }^{a}$ Dang Yuan Lei, ${ }^{\star b}$ Dan Li Wang ${ }^{a}$ and \\ Zhen Xian Huang ${ }^{a}$
}

\begin{abstract}
Optical conductivity plays an important role in characterizing the optoelectronic properties of twodimensional materials. Here we derive the complex optical conductivities for monolayer and few-layered $\mathrm{MoS}_{2}$ films from their reflectance and transmittance responses. We show that the excitonic quantum confinement effect significantly modifies both the peak energy and magnitude of their optical conductivity, manifested by a gradual blueshift in energy (consistent with two well-known models for quantum well systems) and exponential attenuation in magnitude with decreasing layer number. More importantly, the $C$ excition induced optical conductivity peak exhibits the strongest dependence on the $\mathrm{MoS}_{2}$ layer number because of its largest Bohr radius among the A, B and C excitons. This unambiguously confirms the strong influence of quantum confinement effect in the optical conductivity of $\mathrm{MoS}_{2}$, shedding important insights into understanding its rich exciton-related optical properties and therefore facilitating potential applications in optoelectronic devices.
\end{abstract}

\section{Introduction}

In recent years, two-dimensional (2D) transition metal dichalcogenide (TMDC) materials with the generalized formula $\mathrm{MX}_{2}$ $(\mathrm{M}=\mathrm{Mo}, \mathrm{W} ; \mathrm{X}=\mathrm{S}, \mathrm{Se}$, and $\mathrm{Te})$ have received burgeoning research interest due to their peculiar physicochemical properties. ${ }^{1-5}$ Their electronic states generally are subject to strong interlayer coupling and undergo transitions from the indirect bandgap in the bulk form to the direct bandgap in monolayers. In particular, the direct bandgap energy in many monolayer TMDCs lies in the visible and near infrared range, making them ideal candidates for 2D optoelectronic applications. ${ }^{1,2}$ Among various TMDCs, $\mathrm{MoS}_{2}$ is a widely-studied example, which consists of a sandwich structure of S-Mo-S in each layer. ${ }^{2}$ Few-layered $\mathrm{MoS}_{2}$ is an excellent light absorber, with the absorption spectrum generally composed of three characteristic peaks due to excitonic resonance and interband transitions. ${ }^{6}$ In addition, with the abundance of molybdenite in nature, $\mathrm{MoS}_{2}$ is chemically more stable and relatively cheaper than other TMDCs. Previous studies have proven that monolayer $\mathrm{MoS}_{2}$-based photodetectors and phototransistors exhibit high photo-responsivity and a large photo-thermoelectric effect. ${ }^{7,8}$

\footnotetext{
${ }^{a}$ School of Science, Tianjin University of Commerce, Tianjin 300134, PR China. E-mail:gyjia87@163.com

${ }^{b}$ Department of Applied Physics, The Hong Kong Polytechnic University, Hong Kong, PR China. E-mail: dylei@polyu.edu.hk

$\dagger$ Electronic supplementary information (ESI) available: Experimentally measured complex optical conductivity of a monolayer $\mathrm{MoS}_{2}$ film, and calculated thicknessdependent reflectance intensity and magnitude of optical conductivity without considering the excitonic effect. See DOI: 10.1039/c6tc02502a
}

Few-layered $\mathrm{MoS}_{2}$ with thickness-dependent bandgap energy is also promising for fiber lasers, solar cells, optical lenses, gratings, etc. $^{2,9-11}$ In all the aforementioned optoelectronic applications, the optical conductivity of $\mathrm{MoS}_{2}$ plays a key role in characterizing the electronic states of the system and links the current density to an externally applied electric field. ${ }^{12}$

In a typical 2D TMDC material, optical conductivity stems from interband transitions due to electron-photon interaction. When the material absorbs one photon, the generated electron and hole will propagate with the same velocity amplitude but opposite direction, resulting in the so called band nesting. ${ }^{13}$ In the nesting region of the band structure, the conduction and valence bands are parallel to each other in energy. The band nesting gives rise to a singularity in the joint density of states, producing a greatly enhanced optical conductivity. ${ }^{6,13}$ Recently, Wang et al. have successfully measured the photoexcited carrier lifetimes in monolayer and few-layered $\mathrm{MoS}_{2}$ flakes on the basis of frequency-dependent optical conductivity, without carrying out a systematic analysis of the thickness-dependent optical conductivity of $\mathrm{MoS}_{2} \cdot{ }^{14,15}$ Apart from the reports by Wang et al., other research studies on investigating the complex optical conductivity of $\mathrm{MoS}_{2}$ are mainly limited to monolayers. ${ }^{16-18}$ Thus far, the relationship between complex optical conductivity and $\mathrm{MoS}_{2}$ film thickness has not yet been clearly understood. In particular, the detailed influence of the A, B and C excitons on the optical conductivities of monolayer and few-layered $\mathrm{MoS}_{2}$ remains elusive.

In the present work, we calculate the reflectance and transmittance spectra for $\mathrm{MoS}_{2}$ with the number of layers varying from one $(1 \mathrm{~L})$ to ten $(10 \mathrm{~L})$ and subsequently derive the corresponding 
complex optical conductivity and compare the results with the extracted excitonic resonance energies. Our results reveal that the quantum confinement effect in the excitonic resonance has a significant impact on the corresponding induced optical conductivity of $\mathrm{MoS}_{2}$, manifested by different thickness dependences of peak energy and magnitude of optical conductivity peaks associated with different excitonic resonances.

\section{Model system and the theoretical method}

As shown in Fig. 1, our model system consists of a $\mathrm{MoS}_{2}$ film of thickness $L$ deposited on a semi-infinite sapphire substrate. $\mathrm{Yu}$ et al. systematically measured the refractive index $n$ and the extinction coefficient $k$ of atomically thin $\mathrm{MoS}_{2}$ films. They found that both values of $n$ and $k$ are dependent on the film thickness, with the results tabulated in their work and used in our calculation. ${ }^{19}$ In the structure of a multilayer $\mathrm{MoS}_{2}$ film, the Mo atoms of an upper layer locate right above the $\mathrm{S}$ atoms of a lower layer, following an $\mathrm{AB}$ stacking sequence and having the crystal lattice of bulk $2 \mathrm{H}-\mathrm{MoS}_{2} \cdot{ }^{19,20}$ The sapphire substrate is considered as a non-absorbing medium of refractive index $n_{\mathrm{s}}=\sim 1.77$ in the wavelength range of $\lambda=400-900 \mathrm{~nm} .^{21}$ The upper medium of the model system is air and the incident light is perpendicular to the sample surface. The reflectance $R$ and transmittance $T$ of the $\mathrm{MoS}_{2}$ film can then be calculated as ${ }^{18,22}$

$$
g_{2}=\frac{1-n^{2}-k^{2}}{(1+n)^{2}+k^{2}}, \quad h_{2}=\frac{2 k}{(1+n)^{2}+k^{2}}
$$

Note that an alternative approach to calculate the reflectance and transmittance of the $\mathrm{MoS}_{2}$ film involves the use of optical conductivity $\sigma=\sigma_{1}+\mathrm{i} \sigma_{2}$ of $\operatorname{MoS}_{2}$. By Maxwell's equations of the system with appropriate electromagnetic boundary conditions, $R$ and $T$ of the $\mathrm{MoS}_{2}$ film can be expressed as functions of optical conductivity $^{18,23}$

$$
\begin{aligned}
& R=\frac{\left|n_{0}-n_{\mathrm{s}}-\sigma /\left(\varepsilon_{0} c\right)\right|^{2}}{\left|n_{0}+n_{s}+\sigma /\left(\varepsilon_{0} c\right)\right|^{2}} \\
& T=\frac{4 n_{0} n_{\mathrm{s}}}{\left|n_{0}+n_{\mathrm{s}}+\sigma /\left(\varepsilon_{0} c\right)\right|^{2}}
\end{aligned}
$$

where $n_{0}$ stands for the refractive index of the incident medium (here $n_{0}=1.0$ for air), $\varepsilon_{0}$ and $c$ are the free-space permittivity and light velocity, respectively. As a result, one can calculate the thickness-dependent reflectance and transmittance spectra of the $\mathrm{MoS}_{2}$ film by eqn (1) and (2). The real and imaginary parts of $\mathrm{MoS}_{2}$ optical conductivity can then be deduced via solving eqn (3) and (4) with the calculated $R$ and $T$. According to the previous thickness measurement for few-layered $\mathrm{MoS}_{2}$ using an atomic force microscope, ${ }^{19}$ the thicknesses of $1 \mathrm{~L}, 2 \mathrm{~L}, 3 \mathrm{~L}, 4 \mathrm{~L}, 5 \mathrm{~L}$, $6 \mathrm{~L}, 7 \mathrm{~L}, 8 \mathrm{~L}, 9 \mathrm{~L}$ and $10 \mathrm{~L} \mathrm{MoS}_{2}$ films in our calculations are set as $0.65,1.30,1.90,2.50,3.10,3.50,4.30,5.20,5.60$ and $6.20 \mathrm{~nm}$, respectively.

$$
\begin{aligned}
& R=\frac{\left(g_{2}^{2}+h_{2}^{2}\right) \exp (2 \alpha)+\left(g_{1}^{2}+h_{1}^{2}\right) \exp (-2 \alpha)+2\left(g_{1} g_{2}+h_{1} h_{2}\right) \cos 2 \gamma+2\left(g_{2} h_{1}-g_{1} h_{2}\right) \sin 2 \gamma}{\exp (2 \alpha)+\left(g_{1}^{2}+h_{1}^{2}\right)\left(g_{2}^{2}+h_{2}^{2}\right) \exp (-2 \alpha)+2\left(g_{1} g_{2}-h_{1} h_{2}\right) \cos 2 \gamma+2\left(g_{2} h_{1}+g_{1} h_{2}\right) \sin 2 \gamma} \\
& T=\frac{n_{\mathrm{s}}\left[\left(1+g_{1}\right)^{2}+h_{1}^{2}\right]\left[\left(1+g_{2}\right)^{2}+h_{2}^{2}\right]}{\exp (2 \alpha)+\left(g_{1}^{2}+h_{1}^{2}\right)\left(g_{2}^{2}+h_{2}^{2}\right) \exp (-2 \alpha)+2\left(g_{1} g_{2}-h_{1} h_{2}\right) \cos 2 \gamma+2\left(g_{2} h_{1}+g_{1} h_{2}\right) \sin 2 \gamma}
\end{aligned}
$$

where

$$
\begin{gathered}
\alpha=2 \pi k L / \lambda, \quad \gamma=2 \pi n L / \lambda \\
g_{1}=\frac{n^{2}-n_{\mathrm{s}}^{2}+k^{2}}{\left(n_{\mathrm{s}}+n\right)^{2}+k^{2}}, \quad h_{1}=\frac{-2 n_{\mathrm{s}} k}{\left(n_{\mathrm{s}}+n\right)^{2}+k^{2}}
\end{gathered}
$$

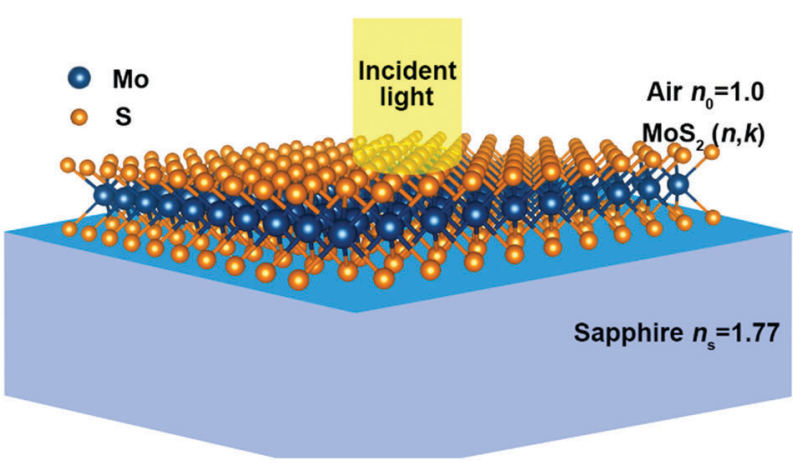

Fig. 1 Schematic depiction of a $\mathrm{MoS}_{2}$ film with the refractive index $n$ and the extinction coefficient $k$. The $\mathrm{MoS}_{2}$ film is deposited on a semi-infinite sapphire substrate.

\section{Results and discussion}

Fig. 2a and $\mathrm{b}$ show the reflectance and transmittance spectra of $\mathrm{MoS}_{2}$ films with various layer numbers. According to energy conservation, the frequency-dependent absorption $A$ of the $\mathrm{MoS}_{2}$ films can also be calculated by $R+T+A=1$. One can clearly see that monolayer $\mathrm{MoS}_{2}$ shows three peaks at 1.879, 2.016 and $2.867 \mathrm{eV}$ in the reflectance spectra (corresponding to dips in the transmittance spectra), which originate respectively from the absorption of A, B and $\mathrm{C}$ excitons. The A and B excitonic peaks come from the direct transitions from two valence bands (which are split due to spin-orbit coupling) to the lowest conduction band at the $K\left(K^{\prime}\right)$ points, while the $\mathrm{C}$ excitonic peak arises from the indirect transition between the valence band maximum located at the $\Gamma$ point and the conduction band minimum located at the $\Lambda$ point of the Brillouin zone. ${ }^{6,19}$ As the layer number increases, the excitonic peaks gradually shift to lower energies. To clearly illustrate the layer dependence, Fig. 3a plots the peak energies of the A, B and C excitons as a function of the $\mathrm{MoS}_{2}$ film thickness. It is estimated that the energy difference between the A and $\mathrm{B}$ excitonic energies is approximately $140 \mathrm{meV}$ for all thicknesses, being an indication of the strength of spin-orbit interaction. 

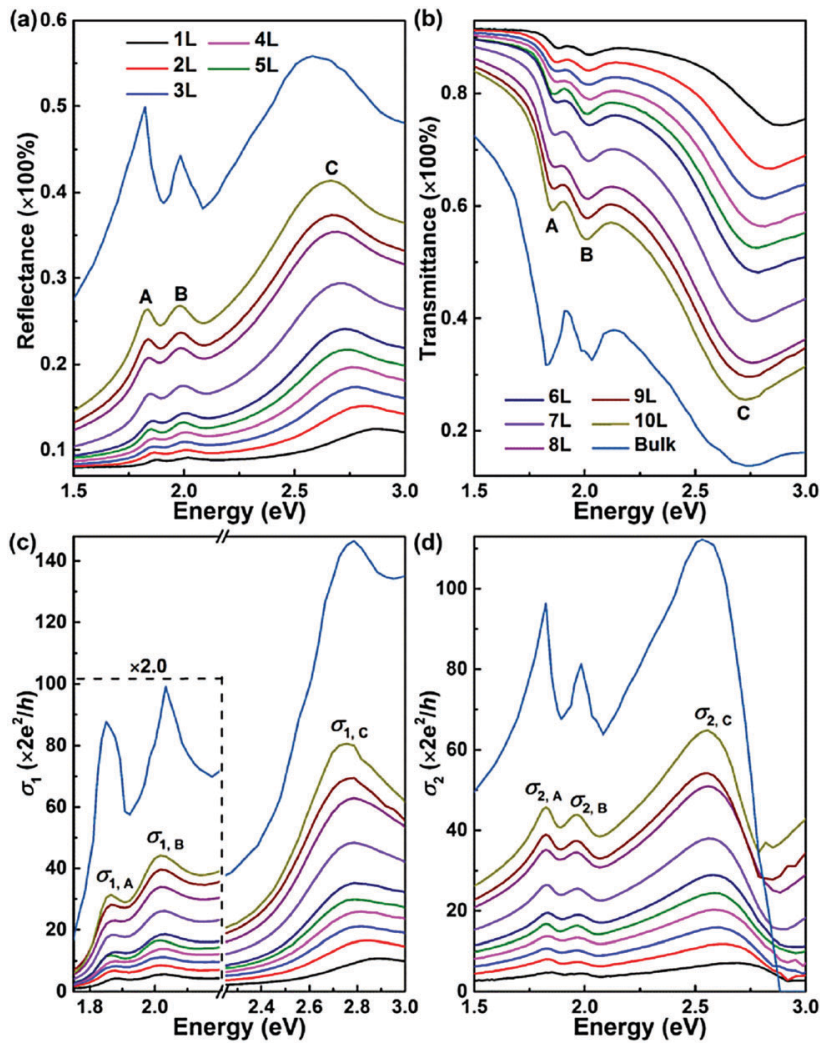

Fig. 2 (a) Reflectance and (b) transmittance spectra, (c) real and (d) imaginary parts of the optical conductivities for $\mathrm{MoS}_{2}$ films with different layer numbers. Legends are given in (a) and (b). Optical conductivity spectra in the dashed box are magnified by a factor of two.

Fig. 2c and d present the real and imaginary parts of the derived optical conductivities for the $\mathrm{MoS}_{2}$ films with various layer numbers. Possibly due to the band nesting effect as mentioned earlier, ${ }^{6,13}$ the $\sigma_{1}\left(\sigma_{2}\right)$ spectra exhibit peaks at energies which are slightly larger (smaller) than the respective A, B and $\mathrm{C}$ excitonic energies. From low to high energies, the three peaks in $\sigma_{1}\left(\sigma_{2}\right)$ spectra are labelled as $\sigma_{1, \mathrm{~A}}, \sigma_{1, \mathrm{~B}}$ and $\sigma_{1, \mathrm{C}}\left(\sigma_{2, \mathrm{~A}}, \sigma_{2, \mathrm{~B}}\right.$ and $\left.\sigma_{2, \mathrm{C}}\right)$, indicating that they are associated with the corresponding excitonic transitions in $\mathrm{MoS}_{2}$, respectively. In our results, both $\sigma_{1}$ and $\sigma_{2}$ are positive, consistent with that reported by Morozov et al. ${ }^{18}$ To further confirm this observation, we measured the reflectance and transmittance of a monolayer $\mathrm{MoS}_{2}$ sample and derived its complex optical conductivity which has positive numbers for both $\sigma_{1}$ and $\sigma_{2}$ (see Fig. S1 in the ESI $\dagger$ ). However, the sign of $\sigma_{2}$ is contrary to the result reported by Wang et al. ${ }^{15}$ The negative $\sigma_{2}$ observed by Wang et al. could partly be attributed to the significantly decreased mobility of charge carriers, because recombination of photoexcited carriers under ultrafast optical pump-probe beams can increase the carrier effective mass and the scattering probability.

Fig. 4a shows the peak energies induced by the A, B and C excitons in the optical conductivity for the $\mathrm{MoS}_{2}$ films with various layer numbers. One can see that the peaks of $\sigma_{1, \mathrm{C}}$ and $\sigma_{2, \mathrm{C}}$ gradually shift towards lower energies as the layer number increases, which appears to mimic the $\mathrm{C}$ excitonic resonance
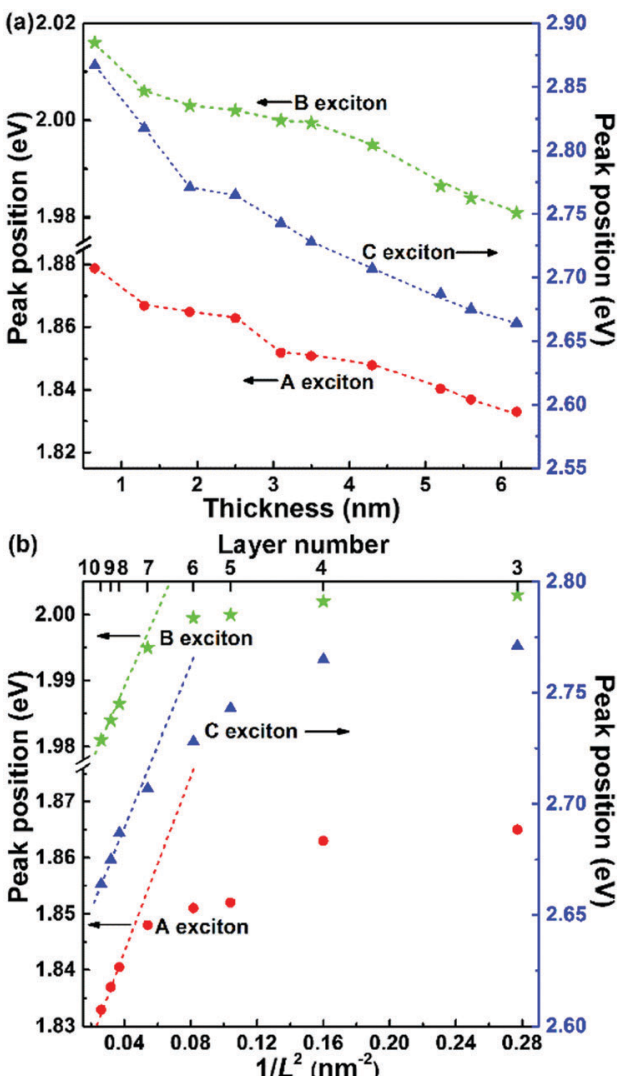

Fig. 3 Peak energies of the $A, B$ and $C$ excitons extracted from the reflectance spectra vs. (a) the $M o S_{2}$ film thickness $L$ and (b) $1 / L^{2}$. Dashed lines in (a) are the fitting results obtained by using eqn (5). Dashed lines in (b) are simulated by using the model of infinite quantum wells.

energies as observed in Fig. 3a. In particular, when the film thickness is larger than $4 \mathrm{~L}$, the energy difference between $\sigma_{1, \mathrm{C}}$ $\left(\sigma_{2, \mathrm{C}}\right)$ and $\mathrm{C}$ excitonic resonance energy exhibits a nearly linear dependence on $L$ as shown in Fig. 4b. However, the peak energies of $\sigma_{1, \mathrm{~A}}$ and $\sigma_{2, \mathrm{~A}}\left(\sigma_{1, \mathrm{~B}}\right.$ and $\left.\sigma_{2, \mathrm{~B}}\right)$ do not exhibit a similar blueshift with decreasing layer number down to $3 \mathrm{~L}(2 \mathrm{~L})$ as shown in Fig. 4a and Table 1. As a result, no such linear dependence of energy difference between the optical conductivity peak and exciton energy on $L$ can be observed for either the A or the B exciton for layer numbers $>3 \mathrm{~L}$ or $2 \mathrm{~L}$. These different effects of the A, B and C excitons in the optical conductivity spectra could be explained by the quantum confinement effect as discussed later.

In the following text, we first evaluate the evolution of excitonic resonance peaks of $\mathrm{MoS}_{2}$ with film thickness, and fit the peak positions extracted from Fig. 2 a by using the models of infinite quantum wells and the quantum wells in fractional dimensional space. ${ }^{19,24,25}$ For the $\mathrm{MoS}_{2}$ films thicker than $7 \mathrm{~L}$, the excitonic peak position $E_{\mathrm{e}}$ linearly depends on $1 / L^{2}$ as presented in Fig. 3b. This matches well with the model of infinite quantum wells, and $E_{\mathrm{e}}$ can be fitted by $E_{\mathrm{e}}=E_{\mathrm{g}}+\pi^{2} \hbar^{2} / 2 \mu L^{2}$, where $E_{\mathrm{g}}$ is the excitonic resonance energy in bulk $\mathrm{MoS}_{2}$ flake $\left(E_{\mathrm{g}}=1.812,1.966\right.$ and $2.616 \mathrm{eV}$ for $\mathrm{A}, \mathrm{B}$ and $\mathrm{C}$ excitons, respectively, according to Fig. $2 \mathrm{a}$ ) and $\mu$ is the reduced electronhole effective mass. The fitting results (dashed lines in Fig. 3b) 

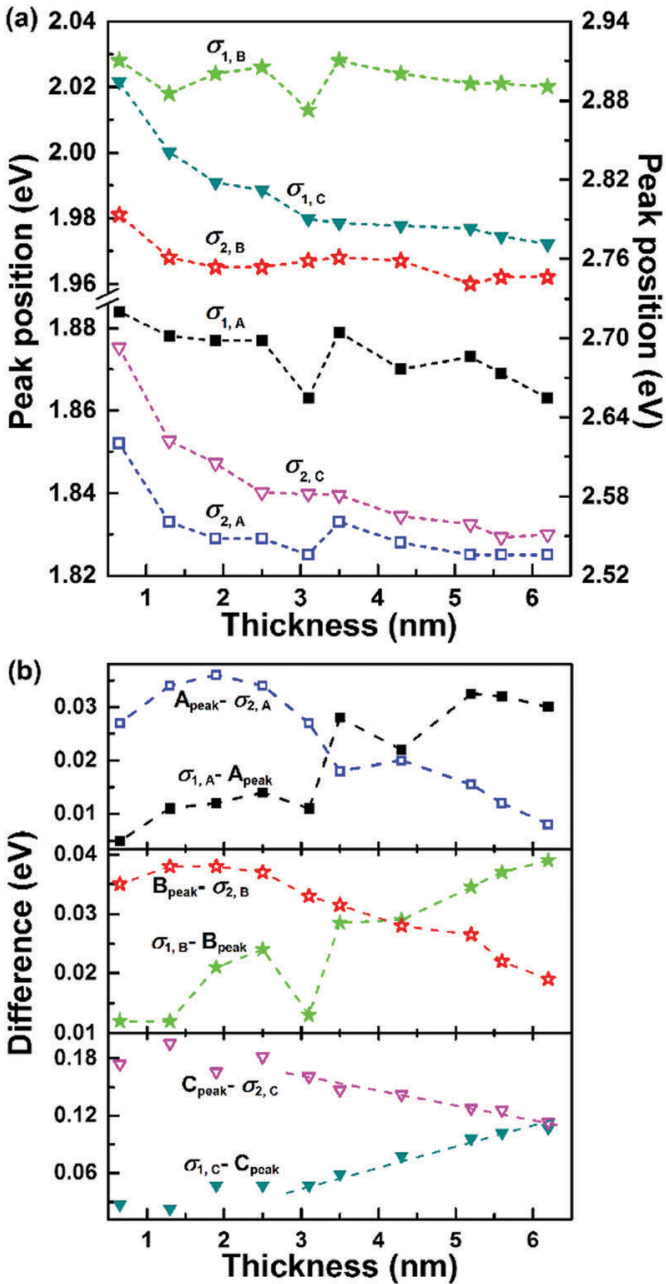

Fig. 4 (a) Excitonic resonance induced peak positions in optical conductivity spectra. Refer to the right (left) vertical axis for $\sigma_{1, \mathrm{C}}$ and $\sigma_{2, \mathrm{c}}$ (the others). (b) Energy differences between optical conductivity peaks and respective excitonic resonance energies. Dashed lines are just drawn as a guide to the eye.

Table 1 Peak positions of optical conductivities in $\mathrm{MoS}_{2}$ films with different layer numbers

\begin{tabular}{lllllll}
\hline \multirow{2}{*}{$\begin{array}{l}\text { Layer } \\
\text { number }\end{array}$} & \multicolumn{6}{l}{ Peak position $(\mathrm{eV})$} \\
\cline { 2 - 7 } & $\sigma_{1, \mathrm{~A}}$ & $\sigma_{2, \mathrm{~A}}$ & $\sigma_{1, \mathrm{~B}}$ & $\sigma_{2, \mathrm{~B}}$ & $\sigma_{1, \mathrm{C}}$ & $\sigma_{2, \mathrm{C}}$ \\
\hline 1 & 1.884 & 1.852 & 2.028 & 1.981 & 2.894 & 2.693 \\
2 & 1.878 & 1.833 & 2.018 & 1.968 & 2.841 & 2.622 \\
3 & 1.877 & 1.829 & 2.024 & 1.965 & 2.818 & 2.605 \\
4 & 1.877 & 1.829 & 2.026 & 1.965 & 2.812 & 2.583 \\
5 & 1.863 & 1.825 & 2.013 & 1.967 & 2.790 & 2.582 \\
6 & 1.879 & 1.833 & 2.028 & 1.968 & 2.787 & 2.581 \\
7 & 1.870 & 1.828 & 2.024 & 1.967 & 2.785 & 2.565 \\
8 & 1.873 & 1.825 & 2.021 & 1.960 & 2.783 & 2.559 \\
9 & 1.869 & 1.825 & 2.021 & 1.962 & 2.777 & 2.549 \\
10 & 1.863 & 1.825 & 2.020 & 1.962 & 2.771 & 2.551 \\
& & & & & &
\end{tabular}

give $E_{\mathrm{e}}=1.812+0.781 / L^{2}, 1.966+0.577 / L^{2}$ and $2.616+1.834 / L^{2}$ for the $\mathrm{A}, \mathrm{B}$ and $\mathrm{C}$ excitons, respectively. With these results we can derive the reduced masses for the A, B and C excitons in $\mathrm{MoS}_{2}$ films thicker than $7 \mathrm{~L}$, which are $\mu=0.482 m_{0}, 0.652 m_{0}$ and $0.205 m_{0}$, respectively.
We can also derive the Bohr radii of excitons by using $a_{\mathrm{B}}=0.0529 m_{0} \varepsilon / \mu$, where $m_{0}$ is the free electron mass and $\varepsilon=10.71$ is the static dielectric constant of bulk $\mathrm{MoS}_{2} \cdot{ }^{26}$ The Bohr radii of A, $\mathrm{B}$ and $\mathrm{C}$ excitons are calculated to be $1.177,0.869$ and $2.763 \mathrm{~nm}$, respectively. Since in the literature it lacks a comprehensive comparison about the physical parameters of the $\mathrm{A}, \mathrm{B}$ and $\mathrm{C}$ excitons in $\mathrm{MoS}_{2}$ films, it may be difficult to thoroughly crosscheck all our calculated results. However, the derived $\mu$ values for the $\mathrm{A}$ and $\mathrm{C}$ excitons are reasonably in line with previously reported results which are $0.42 m_{0}$ and $0.25 m_{0}$ for bulk $\mathrm{MoS}_{2}$, respectively. ${ }^{19,27}$ Here the derived Bohr radii in thick/bulk $\mathrm{MoS}_{2}$ are also comparable with the previous experimental results which are $\sim 2.0$ and $\sim 0.8 \mathrm{~nm}$ for the $\mathrm{A}$ and $\mathrm{B}$ excitons, respectively. ${ }^{28,29}$

For $\mathrm{MoS}_{2}$ films with layer numbers $\leq 7 \mathrm{~L}$, the excitonic peak positions deviate from the model of infinite quantum wells (see Fig. 3b). Instead, they could be fitted by the model of confined quantum wells with ${ }^{19,24,25}$

$$
E_{\mathrm{e}}=E_{\mathrm{g}}+R_{\mathrm{b}}+\frac{\pi^{2} \hbar^{2}}{2 \mu L^{2}}[(\alpha-1) / 2]^{2}-\frac{R_{\mathrm{b}}}{[(\alpha-1) / 2]^{2}}
$$

where $R_{\mathrm{b}}=13.6 \mu / m_{0} \varepsilon^{2}$ is the exciton binding energy in bulk $\mathrm{MoS}_{2}$ and $\alpha$ is a parameter to describe the dimensionality of the confined exciton. By using eqn (5) to fit the excitonic peak positions in Fig. 3a, we can get the effective dimensionality $\alpha$ for the A, B and C excitons in $1 \mathrm{~L} \sim 7 \mathrm{~L} \mathrm{MoS}$ films, as shown in Table 2 . It is seen that the $\alpha$ value gradually increases from $\sim 2$ for $1 \mathrm{~L}$ to 3 for $8 \mathrm{~L}$, reasonably depicting the evolution of $\mathrm{MoS}_{2}$ films from the $2 \mathrm{D}$ to $3 \mathrm{D}$ form. When $\alpha$ equals to 3 for layer numbers $\geq 8 \mathrm{~L}$, eqn (5) is naturally reduced to the equation for infinite quantum wells.

Using only the imaginary part of the dielectric function of $\mathrm{MoS}_{2}$, Yu et al. quantitatively fitted the peak position of the $\mathrm{C}$ exciton and extracted the related physical parameters as a function of the layer number. ${ }^{19}$ Nevertheless, they encountered bottleneck problems when dealing with the A and B excitons due to the fact that the peak positions of the A and B excitons exhibit no substantial layer dependence in the dielectric function. Interestingly, by substituting their measured dielectric functions into eqn (1), the calculated reflectance spectra (Fig. 2a) show that all the peaks of the $\mathrm{A}, \mathrm{B}$ and $\mathrm{C}$ excitons gradually shift to lower energies with increasing layer number. Although the underlying physics for this observation needs further investigation, our calculation method can be used to extract the relevant physical

Table 2 Effective dimensionalities $\alpha$ and Bohr radii $a_{\mathrm{B}}$ of excitons in different layer numbers of $\mathrm{MoS}_{2}$ films

\begin{tabular}{|c|c|c|c|c|c|c|}
\hline \multirow{2}{*}{$\begin{array}{l}\text { Layer } \\
\text { number }\end{array}$} & \multicolumn{3}{|l|}{$\underline{\alpha}$} & \multicolumn{3}{|l|}{$\underline{a_{\mathrm{B}}(\mathrm{nm})}$} \\
\hline & A exciton & B exciton & C exciton & A exciton & B exciton & C exciton \\
\hline 1 & 1.845 & 1.955 & 1.649 & 0.497 & 0.415 & 0.897 \\
\hline 2 & 2.182 & 2.303 & 2.005 & 0.695 & 0.566 & 1.389 \\
\hline 3 & 2.420 & 2.524 & 2.237 & 0.836 & 0.662 & 1.709 \\
\hline 4 & 2.615 & 2.695 & 2.510 & 0.950 & 0.737 & 2.086 \\
\hline 5 & 2.720 & 2.822 & 2.692 & 1.012 & 0.792 & 2.338 \\
\hline 6 & 2.806 & 2.895 & 2.780 & 1.063 & 0.824 & 2.459 \\
\hline 7 & 2.939 & 2.980 & 2.935 & 1.141 & 0.861 & 2.673 \\
\hline$\geq 8$ & 3.000 & 3.000 & 3.000 & 1.177 & 0.869 & 2.763 \\
\hline
\end{tabular}


parameters of the $\mathrm{A}, \mathrm{B}$ and $\mathrm{C}$ excitons, and open up the opportunity to have a comprehensive comparison between them. For example, by using the $\alpha$ values given in Table 2, one can derive the effective exciton binding energy from $R_{\mathrm{b}}{ }^{\prime}=4 R_{\mathrm{b}} /(\alpha-1)^{2} .^{25}$ Additionally, the effective Bohr radii of the excitons can also be obtained by utilizing $a_{\mathrm{B}}{ }^{\prime}=a_{\mathrm{B}}(\alpha-1) / 2,{ }^{25}$ as shown in Table 2 .

Here we should point out that there are strong interface couplings, e.g., van der Waals interaction, in the few-layered $\mathrm{MoS}_{2}$ and between the monolayer $\mathrm{MoS}_{2}$ and the substrate. Moreover, the functional form of electron-hole interaction could be modified by the nonlocal nature of dielectric screening. ${ }^{30,31}$ Thus the borrowing of eqn (5) which assumes a homogeneous quantum well to $2 \mathrm{D}$ materials is essentially an approximation. This may also be the reason why the values of $\alpha$ in Table 2 for the three excitons of the monolayer $\mathrm{MoS}_{2}$ are smaller than 2. By using the dielectric screened hydrogen model, ${ }^{30,31}$ the effective binding energy of the $\mathrm{C}$ exciton in the monolayer $\mathrm{MoS}_{2}$ is calculated to be $0.72 \mathrm{eV}$. Substituting this value instead of $R_{\mathrm{b}}{ }^{\prime}$ into eqn (5), we deduce the value of $\alpha$ to be 1.934. It is clear that the incorporation of a dielectric screening effect makes a more reasonable value for $\alpha$. In order to obtain an accurate dimensionality, taking into account the interface couplings as well as the effect of dielectric screening on the confinement energy [the third term in eqn (5)] may be necessary. This may need more complicated theoretical studies. Even so, we believe that the simplicity is the merit of eqn (5). Besides, the model of confined quantum wells leaves out many other influence factors, making it feasible to solely inspect the quantum confinement effect.

Fig. 5 shows the extracted Bohr radii $a_{\mathrm{B}}{ }^{\prime}$ as a function of the film thickness. One can see that the $\mathrm{C}$ exciton has the largest Bohr radius while the $\mathrm{B}$ exciton gives the smallest size in the same thickness of $\mathrm{MoS}_{2}$ film. Furthermore, the Bohr radius of the $\mathrm{C}$ exciton rapidly decreases with reducing thickness after the film thins to $7 \mathrm{~L}$, whereas, the decreasing rates of the A and $\mathrm{B}$ excitonic radii are relatively slower than $\mathrm{C}$. In a semiconductor crystal, an exciton will experience strong or weak quantum confinement when the semiconductor thickness is less or greater than double the Bohr radius of the exciton. ${ }^{32}$ The sizes of the A,

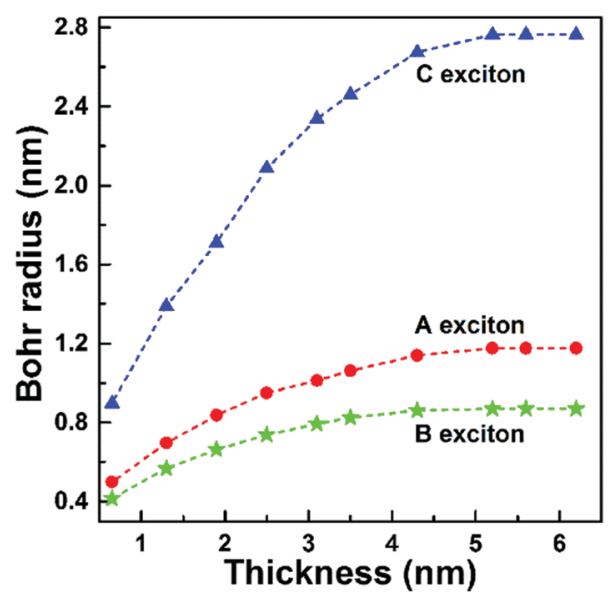

Fig. 5 Bohr radii of A, B and C excitons vs. the $\mathrm{MoS}_{2}$ film thickness. Dashed lines are just drawn as a guide to the eye.
$\mathrm{B}$ and $\mathrm{C}$ excitons in the thick/bulk $\mathrm{MoS}_{2}$ are $2 a_{\mathrm{B}}=\sim 2.35, \sim 1.74$ and $\sim 5.53 \mathrm{~nm}$, respectively. They are close to the thicknesses of $3 \mathrm{~L}, 2 \mathrm{~L}$ and $8 \mathrm{~L} \mathrm{MoS}_{2}$ films, i.e., 1.90, 1.30 and $5.20 \mathrm{~nm}$, respectively. As a consequence, the $\mathrm{A}, \mathrm{B}$ and $\mathrm{C}$ excitons in thin $\mathrm{MoS}_{2}$ films are expected to experience a strong quantum confinement effect. However, the $\mathrm{C}$ exciton is more tightly confined in most of the films studied in our work while the A and B excitons can be confined only in films thinner than $4 \mathrm{~L}$ and $3 \mathrm{~L}$, respectively. The variation in the $\mathrm{C}$ excitonic radius presents the largest range as the layer number decreases to $1 \mathrm{~L}$ (see Fig. 5), implying that the excitonic quantum confinement plays the strongest effect on the $\mathrm{C}$ exciton in comparison with the $\mathrm{A}$ and B excitons. As a result, the peaks of $\sigma_{1, \mathrm{C}}$ and $\sigma_{2, \mathrm{C}}$ gradually shift towards lower energies with increasing layer number (Fig. 4a) and the energy difference between the $\sigma_{1, \mathrm{C}}\left(\sigma_{2, \mathrm{C}}\right)$ peak and $\mathrm{C}$ excitonic resonance energy linearly depends on the thickness for films thicker than $4 \mathrm{~L}$ (see Fig. $4 \mathrm{~b}$ ). In sharp contrast, the peak positions of $\sigma_{1, \mathrm{~A}}$ and $\sigma_{2, \mathrm{~A}}\left(\sigma_{1, \mathrm{~B}}\right.$ and $\left.\sigma_{2, \mathrm{~B}}\right)$ fluctuate with the layer number when the $\mathrm{MoS}_{2}$ film is thicker than $3 \mathrm{~L}(2 \mathrm{~L})$, as shown in Fig. 4. Note that the fluctuation of the energy difference $\sigma_{1, \mathrm{C} \text {,peak }}-\mathrm{C}_{\text {peak }}\left(\right.$ or $\mathrm{C}_{\text {peak }}-\sigma_{2, \mathrm{C} \text {,peak }}$ ) in films thinner than $5 \mathrm{~L}$ could be a result of substrate-induced van der Waals interaction and/or the local doping effect. ${ }^{33,34}$ Previous studies have shown that the energy variation in the excitonic peak position induced by the influence of the substrate could be up to $\sim 80 \mathrm{meV}$ for a bilayer $\mathrm{MoS}_{2}$ film, and this kind of influence decreases significantly with increasing the layer number. ${ }^{33,34}$

In addition to the effect in the peak position of $\mathrm{MoS}_{2}$ optical conductivity, our results further show that the excitonic quantum confinement phenomenon also has a strong influence in the excitonic peak intensity and the magnitudes of $\sigma_{1, \mathrm{~A}}, \sigma_{1, \mathrm{~B}}$ and $\sigma_{1, \mathrm{C}}\left(\sigma_{2, \mathrm{~A}}, \sigma_{2, \mathrm{~B}}\right.$ and $\left.\sigma_{2, \mathrm{C}}\right)$. Intuitively, it is reasonable that the reflectance intensity and the optical conductivity magnitude decrease upon reducing the $\mathrm{MoS}_{2}$ film thickness. By using the measured dielectric function, e.g., the $n$ and $k$ values for bulk $\mathrm{MoS}_{2}$, the calculation results (see Fig. S2 in the ESI $\dagger$ ) show that both reflectance intensity and optical conductivity decrease
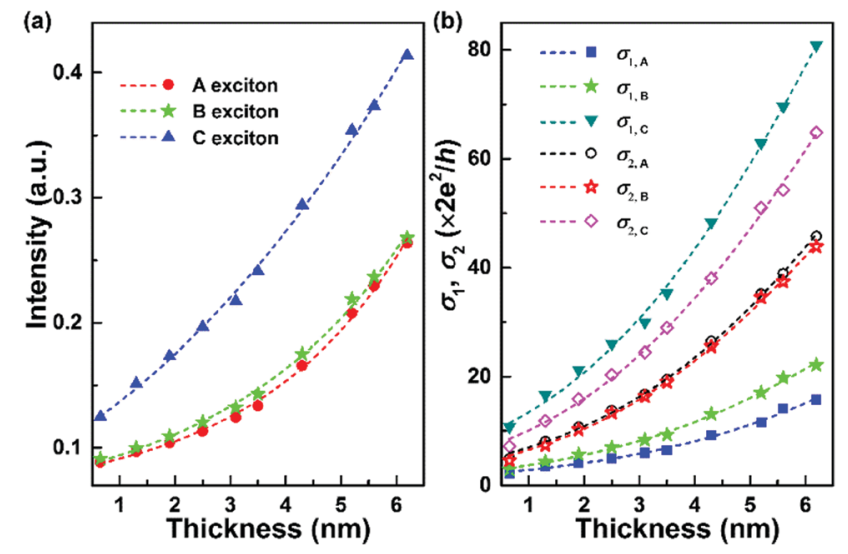

Fig. 6 (a) Reflectance intensities and (b) magnitudes of optical conductivity induced by $\mathrm{A}, \mathrm{B}$ and $\mathrm{C}$ excitons vs. the $\mathrm{MoS}_{2}$ film thickness. Symbols are the results extracted from Fig. 2. Dashed lines are the fitting results obtained by using eqn (6). 
Table 3 Detailed values of fitting coefficients $a, b$ and $c$ in eqn (6)

\begin{tabular}{|c|c|c|c|c|c|c|c|c|c|}
\hline & \multicolumn{3}{|c|}{ Reflectance } & \multicolumn{6}{|c|}{ Optical conductivity } \\
\hline & A exciton & B exciton & C exciton & $\sigma_{1, \mathrm{~A}}$ & $\sigma_{2, \mathrm{~A}}$ & $\sigma_{1, \mathrm{~B}}$ & $\sigma_{2, \mathrm{~B}}$ & $\sigma_{1, \mathrm{C}}$ & $\sigma_{2, \mathrm{C}}$ \\
\hline$a$ & -2.5056 & -2.5100 & -2.2470 & 0.6572 & 1.4621 & 0.8487 & 1.3611 & 2.1269 & 1.8121 \\
\hline$b$ & 0.0961 & 0.1366 & 0.2663 & 0.3895 & 0.4992 & 0.4653 & 0.5355 & 0.4945 & 0.5252 \\
\hline$c$ & 0.0154 & 0.0094 & -0.0073 & 0.0077 & -0.0189 & -0.0160 & -0.0232 & -0.0208 & -0.0234 \\
\hline
\end{tabular}

linearly upon decreasing the film thickness. By contrast, if we take into account the excitonic quantum confinement effect, we find that both of them induced by the A, B and C excitons, as shown in Fig. 6, are attenuated by reducing the film thickness, following an exponential function

$$
I=\exp \left(a+b L+c L^{2}\right)
$$

where $I$ stands for the intensity or magnitude, $a, b$ and $c$ are fitting coefficients with detailed values given in Table 3. Comparing the results in Fig. 6 and Fig. S2 (ESI†) reveals that the excitonic quantum confinement effect significantly accelerates the decay of the exciton-induced reflectance peak intensity and the optical conductivity peak magnitude for $\mathrm{MoS}_{2}$ of the same thickness. Since most of the previous studies on 2D TMDCs were limited to the film with the maximum layer number of $5 \mathrm{~L}$, they failed to give a useful empirical formula to predict the peak magnitude of optical conductivity. Eqn (6) and Table 3 may provide guidelines to the evolution of the optoelectronic properties of the A, $\mathrm{B}$ and C excitons with $\mathrm{MoS}_{2}$ film thickness.

\section{Conclusions}

In summary, the layer-dependent optical conductivities of $\mathrm{MoS}_{2}$ films have been derived from their calculated reflectance and transmittance spectra in the visible to near-infrared region. It is found that the $\mathrm{C}$ exciton has the largest Bohr radius in the same thickness of $\mathrm{MoS}_{2}$ film such that it is subjected to the strongest quantum confinement effect compared with the $\mathrm{A}$ and $\mathrm{B}$ excitons. As a result, both peak positions of real and imaginary parts of the optical conductivity induced by the $\mathrm{C}$ exciton gradually shift towards high energies with decreasing film thickness. In remarkable contrast, this blueshift tendency is not observed for either the A or the B exciton for films thicker than $3 \mathrm{~L}$ or $2 \mathrm{~L}$, respectively. Additionally, our results reveal that the attenuations of reflectance intensity and the magnitude of optical conductivity induced by the A, B and C excitons follow an exponential decaying function with decreasing $\mathrm{MoS}_{2}$ film thickness. These findings may provide important insights into the critical role of the excitonic quantum confinement effect in the photoelectric properties of 2D TMDCs in general. The fitting equations along with the derived physical parameters may also provide useful guidelines for future applications of $\mathrm{MoS}_{2}$ materials in 2D nanodevice fabrications.

\section{Acknowledgements}

We gratefully acknowledge Dr Yiling Yu and Prof. Linyou Cao from North Carolina State University who shared their measured optical constants of bulk $\mathrm{MoS}_{2}$. We are also grateful to Mr Chang Qin from 6Carbon Technology for his help with the preparation of the monolayer $\mathrm{MoS}_{2}$ film on the sapphire substrate. We acknowledge the financial support from the Hong Kong Polytechnic University (Grant No. 1-ZVCG).

\section{Notes and references}

1 G. R. Bhimanapati, Z. Lin, V. Meunier, Y. Jung, J. Cha, S. Das, D. Xiao, Y. Son, M. S. Strano, V. R. Cooper, L. Liang, S. G. Louie, E. Ringe, W. Zhou, S. S. Kim, R. R. Naik, B. G. Sumpter, H. Terrones, F. Xia, Y. Wang, J. Zhu, D. Akinwande, N. Alem, J. A. Schuller, R. E. Schaak, M. Terrones and J. A. Robinson, ACS Nano, 2015, 9, 11509-11539.

2 R. Ganatra and Q. Zhang, ACS Nano, 2014, 8, 4074-4099.

3 X. D. Li, S. Q. Wu and Z. Z. Zhu, J. Mater. Chem. C, 2015, 3, 9403-9411.

4 X. H. Wang, J. Q. Ning, C. C. Zheng, B. R. Zhu, L. Xie, H. S. Wu and S. J. Xu, J. Mater. Chem. C, 2015, 3, 2589-2592.

5 Y. Li, N. Dong, S. Zhang, K. Wang, L. Zhang and J. Wang, Nanoscale, 2016, 8, 1210-1215.

6 D. Kozawa, R. Kumar, A. Carvalho, K. K. Amara, W. Zhao, S. Wang, M. Toh, R. M. Ribeiro, A. H. Castro Neto, K. Matsuda and G. Eda, Nat. Commun., 2014, 5, 4543.

7 Y. C. Wu, C. H. Liu, S. Y. Chen, F. Y. Shih, P. H. Ho, C. W. Chen, C. T. Liang and W. H. Wang, Sci. Rep., 2015, 5, 11472.

8 N. Perea-López, Z. Lin, N. R. Pradhan, A. Iñiguez-Rábago, A. L. Elías, A. McCreary, J. Lou, P. M. Ajayan, H. Terrones, L. Balicas and M. Terrones, 2D Mater., 2014, 1, 011004.

9 P. Li, B. Liang, M. Su, Y. Zhang, Y. Zhao, M. Zhang, C. Ma and N. Su, Appl. Phys. B: Lasers Opt., 2016, 122, 150.

10 Y. G. Kim, K. C. Kwon, Q. V. Le, K. Hong, H. W. Jang and S. Y. Kim, J. Power Sources, 2016, 319, 1-8.

11 J. Yang, Z. Wang, F. Wang, R. Xu, J. Tao, S. Zhang, Q. Qin, B. Luther-Davies, C. Jagadish, Z. Yu and Y. Lu, Light: Sci. Appl., 2016, 5, e16046.

12 Z. Wu, E. Taylor and E. Zaremba, Europhys. Lett., 2015, 110, 26002.

13 A. Carvalho, R. M. Ribeiro and A. H. Castro Neto, Phys. Rev. B: Condens. Matter Mater. Phys., 2013, 88, 115205.

14 H. Wang, C. Zhang and F. Rana, Nano Lett., 2015, 15, 339-345.

15 H. Wang, C. Zhang and F. Rana, Nano Lett., 2015, 15, 8204-8210.

16 Z. Li and J. P. Carbotte, Phys. Rev. B: Condens. Matter Mater. Phys., 2012, 86, 205425. 
17 H. Rostami and R. Asgari, Phys. Rev. B: Condens. Matter Mater. Phys., 2014, 89, 115413.

18 Y. V. Morozov and M. Kuno, Appl. Phys. Lett., 2015, 107, 083103.

19 Y. Yu, Y. Yu, Y. Cai, W. Li, A. Gurarslan, H. Peelaers, D. E. Aspnes, C. G. Van de Walle, N. V. Nguyen, Y.-W. Zhang and L. Cao, Sci. Rep., 2015, 5, 16996.

20 Y. Yu, C. Li, Y. Liu, L. Su, Y. Zhang and L. Cao, Sci. Rep., 2013, 3, 1866.

21 Refractive index database: http://refractiveindex.info/?shelf= main\&book=Al2O3\&page=Malitson-o.

22 O. S. Heavens, Rep. Prog. Phys., 1960, 23, 1-65.

23 T. Stauber, N. M. R. Peres and A. K. Geim, Phys. Rev. B: Condens. Matter Mater. Phys., 2008, 78, 085432.

24 X. F. He, Phys. Rev. B: Condens. Matter Mater. Phys., 1990, 42, 11751-11756.

25 X. F. He, Phys. Rev. B: Condens. Matter Mater. Phys., 1991, 43, 2063-2069.
26 A. Thilagam, J. Appl. Phys., 2014, 116, 053523.

27 E. Fortin and F. Raga, Phys. Rev. B: Condens. Matter Mater. Phys., 1975, 11, 905-912.

28 R. F. Frindt, Phys. Rev., 1965, 140, 536-539.

29 T. Sekine, K. Uchinokura, T. Nakashizu, E. Matsuura and R. Yoshizaki, J. Phys. Soc. Jpn., 1984, 53, 811-818.

30 T. Olsen, S. Latini, F. Rasmussen and K. S. Thygesen, Phys. Rev. Lett., 2016, 116, 056401.

31 S. Latini, T. Olsen and K. S. Thygesen, Phys. Rev. B: Condens. Matter Mater. Phys., 2015, 92, 245123.

32 D. W. Snoke, Solid State Physics: Essential Concepts, Pearson/ Addison-Wesley, 2009.

33 M. Buscema, G. A. Steele, H. S. J. van der Zant and A. Castellanos-Gomez, Nano Res., 2014, 7, 561-571.

34 N. Scheuschner, O. Ochedowski, A.-M. Kaulitz, R. Gillen, M. Schleberger and J. Maultzsch, Phys. Rev. B: Condens. Matter Mater. Phys., 2014, 89, 125406. 